\title{
Some recent advances in bulk growth of mercury cadmium telluride crystals
}

\author{
R K SHARMA, R K BAGAI and VIKRAM KUMAR \\ Solid State Physics Laboratory, Lucknow Road, Delhi 110054, India
}

\begin{abstract}
The inherent metallurgical problems associated with the $\mathrm{HgTe} / \mathrm{CdTe}$ pseudobinary alloy system render the standard crystal growth processes inapplicable to the preparation of mercury cadmium telluride crystals for infrared detector applications. A variety of rather nonconventional techniques have been developed to overcome these problems. Two such techniques, viz. asymmetrical Bridgman and horizontal casting for solid-state recrystallization, developed at Solid State Physics Laboratory for the bulk growth of mercury cadmium telluride crystals are reviewed in this communication.

Due to the poor thermal conductivity of mercury cadmium telluride melts and solids, and the use of thick-walled quartz ampuoles, it is extremely difficult to obtain a flat solid-liquid interface during Bridgman growth of this material. The technique of asymmetrical Bridgman has been successful in overcoming this problem to a great extent. Solid-state recrystallization has been widely accepted as one of the most successful techniques for obtaining large quantities of acceptable-quality mercury cadmium telluride crystals for infrared detector applications. This is a two-step process - the melt is first quenched to obtain a good cast, which is then subjected to a grain-growth annealing. The horizontal casting procedure developed for solid state recrystallization growth has been successful in improving the overall quality and yield of bulk mercury cadmium telluride crystals.
\end{abstract}

Keywords. Mercury cadmium telluride crystals; bulk growth.

\section{Introduction}

Solid-state electronic devices have played a vital role in the scientific and technological advancements of the later part of the current century. Particularly, the fields of electronics, optoelectronics and, lately, photonics were revolutionized with the development of some specific devices. Many of these devices could be realized, solely due to the invention of some specific materials. The discovery and development of new materials and devices has, in fact, been a regular feature of applied scientific research throughout these years. The materials of interest include semiconductors, insulators, ferrites, electrets, superconductors, etc which are used either as bulk crystals, or as epitaxial crystalline/strained-layered structures, quantum/nano-structures, etc for fabricating specific devices/device-structures. The stringent requirements of specific materials with desired characteristics for individual device fabrication make it imperative to have both material and device R\&D works to be undertaken side by side. Major establishments engaged in device development work do, therefore, have material activities also going on concurrently. Solid State Physics Laboratory (SSPL) too is engaged in an extensive materials research activity to back up the widespread R\&D work on advanced devices/device-structures. Figure 1 summarizes the materials activity at SSPL, which is supported by a range of material preparation and characterization facilities. The materials under study include semiconductors, oxide crystals and ferrites used for a variety of advanced electronic and optoelectronic devices. The material preparation techniques include bulk as well as epitaxial processes, including liquid-phase epitaxy (LPE), metal organic chemical vapour deposition (MOCVD), 


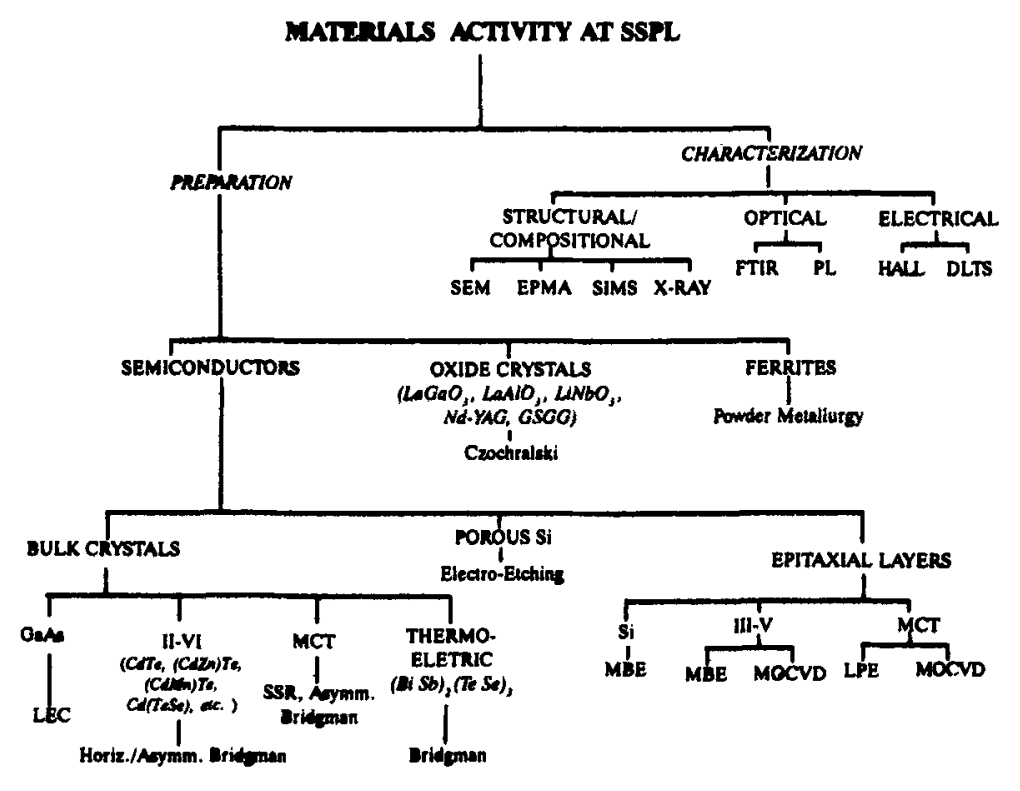

Figue 1. Materials activity at Solid State Physics Laboratory.

molecular beam epitaxy (MDE), etc. The characterization facilities include those for optical and scanning electron microscopy (SEM), electron probe microanalysis (EPMA), secondary ion mass spectrometry (SIMS), X-ray diffraction/topography, etc for structural/microstructural/compositional evaluation; Fourier-transform infrared (FTIR) and photoluminescence (PL) spectrometry for optical evaluation; and lowtemperature Hall, deep-level transient spectrometry (DLTS), etc for electrical characterization. The prominent among the materials under study are various semiconductor materials including III-V, II-VI and other compounds/alloys, in addition to certain oxide crystals and ferrites.

Considerable progress in the preparation and characterization of these materials in the form of bulk crystals as well as epitaxial layers, including quantum well structures, etc has been made at SSPL as a result of consistent R\&D work. However, the scope of this review is restricted to the preparation of bulk crystals of narrow-band gap alloy semiconductor mercury cadmium telluride (MCT, $\mathrm{Hg}_{1-x} \mathrm{Cd}_{x} \mathrm{Te}$ ) (Cruse 1981), which is one of the most extensively studied materials here.

MCT is an alloy of two II-VI compounds, HgTe and CdTe, which are miscible in all proportions. Its band gap varies almost linearly with composition between those of semimetallic $\mathrm{Hg} \mathrm{Te}(-0.3 \mathrm{eV})$ and semiconducting $\mathrm{CdTe}(1.6 \mathrm{eV})$. The $\mathrm{Hg}$-rich compositions of this alloy are narrow-gap semiconductors for $x>0.16$, and are, therefore, useful for IR detection over $a$ wide wavelength range. However, the compositions with $x \sim 0.2$ are of most significance, as they have a band gap $\sim 0.1 \mathrm{eV}$, suitable for detecting IR radiation emitted by objects at around ambient temperature and transmitted through the strategically important $8-14 \mu \mathrm{m}$ atmospheric window (Elliot and Gordon 1993). Due to some unique intrinsic characteristics like high optical absorption due to a direct band gap, very low electron effective mass $\left(\sim 0.005 \mathrm{~m}_{0}\right)$, high electron mobility $\left(\sim 2 \times 10^{5} \mathrm{~cm}^{2} \mathrm{~V}^{-1} \mathrm{~s}^{-1}\right)$, low dielectric constant $\left(\varepsilon_{\mathrm{r}} \sim 16\right)$, etc MCT 
has proved to be the most successful material for fabricating IR detectors for strategic and commercial applications (Proc. SPIE 1979).

Although both bulk and epitaxial crystals of MCT are utilized for fabricating photoconductive (PC) and photovoltaic (PV) detectors, bulk-crystal-based linear PC and epitaxial-layer-based planar PV arrays are the most commonly used configurations. However, the preparation of device-quality bulk as well as epitaxial MCT crystals is a difficult task due to a large number of inherent metallurgical complexities associated with $\mathrm{Hg}-\mathrm{Cd}-\mathrm{Te}$ pseudobinary alloy system (Sharma and Sharma 1987), and some specific techniques are required to be evolved/perfected to overcome these problems. In the present paper we review the indigenously developed techniques of asymmetrical Bridgman and horizontal casting for solid state recrystallization (SSR) growth of bulk MCT crystals.

\section{Bulk growth of MCT}

The inherent metallurgical problems, mainly a wide phase diagram and very high $\mathrm{Hg}$ vapour pressures, associated with $\mathrm{HgTe} / \mathrm{CdTe}$ pseudobinary alloy system render the conventional melt growth techniques inapplicable, as such, to MCT bulk growth. The $\mathrm{Hg}$ vapour pressure over the stoichiometric $\mathrm{MCT}$ melts at the liquidus point is $\sim 35$ atm. Due to this, growth of MCT crystals from melts is required to be carried out in closed systems, such as sealed thick-walled $(\sim 2.5-3 \mathrm{~mm})$ quartz containers. The Czochralski growth process, which is currently the most successful one in case of $\mathrm{Si}$ and GaAs, cannot at all be applied to MCT. Moreover, the larga CdTe segregation in the solid phase during freezing of melt $\left(c_{\mathrm{s}} / c_{1} \sim 3\right)$, as a consequence of the broad pseudobinary phase diagram, requires the use of high temperature gradients to avoid constitutional supercooling across the solid liquid $(\mathrm{s}-1)$ interface during Bridgman growth of MCT crystals. The s-1 interface normally remains quite curved due to the poor thermal conductivity of MCT melts and solids, in addition to the adverse effects of thick quartz walls. The heat is virtually extracted from the peripheral regions through radiation with negligible contribution from the solidified material and the melt. This results in marked compositional non-uniformity, both radial as well as axial, in MCT ingots grown conventionally using Bridgman method. The radial compositional variation due to peripheral segregation of CdTe also imposes a maximum limit to the ingot diameter (usually $<12 \mathrm{~mm}$ ). An improvement in compositional uniformity of such ingots can be obtained with the use of accelerated crucible rotation technique (ACRT) (Capper et al 1988). However, this involves the use of sophisticated instrumentation in addition to a sustained standardization schedule. An innovative modification of conventional Bridgman method, called asymmetrical Bridgman, was suggested by Bagai and Borle (1989) at SSPL, which resulted in marked improvement in the compositional uniformity by way of making $\mathrm{s}-1$ interface planar. This method is further discussed later in this section.

A variety of rather nonconventional techniques for bulk growth of MCT also emerged as a result of consistent efforts to circumvent the above problems. Solid-state recrystallization (SSR) (Micklethwaite 1981), variously called cast recrystallize anneal (CRA) or cast quench anneal (CQA), is one of the earliest, most successful and widely employed techniques among them. This process has little analogy with the SSR process in metal systems, as the latter follows a cold work for nucleation of a fine-grained 
microstructure. Here a good cast is first obtained by quenching or by controlled cooling of MCT melt, which is then kept for a long-term (3-5 weeks) annealing just near the solidus temperature $\left(660-670^{\circ} \mathrm{C}\right)$ for the grain growth. Large-sized singlecrystalline grains are normally obtained after the second step. The critical step in this process is the initial casting which controls the overall compositional uniformity of the ingot. With proper casting of ingots through appropriate quenching procedure and, finally, with selective slicing of grown ingots, large crystals, with much better compositional uniformity compared to conventional Bridgman ingots, could be obtained. However, the quenching efficiency of MCT ingots is normally poor due to the use of thick-walled quartz ampoules and poor thermal conductivity of the melts. This again puts a constraint on the maximum ingot diameter. The urge to improve the ingot size led to the development of various variants of SSR by different groups of workers. The most important of them are pressurized CRA (also called RSRE CRA) (Vere et al 1982) and incremental quenching (Luigi Colombo et al 1985). However, both these methods involve much technical complexity. A relatively simple method of horizontal casting was invented by Sharma and co-workers (1993) at SSPL. This method not only resulted in a marked improvement in the compositional uniformity of SSR-grown MCT crystals but also crystalline perfection was improved to a great extent. These two techniques, asymmetrical Bridgman and horizontal casting, which are quite successful in obtaining bulk MCT crystals for device fabrication, are now discussed in a little detail.

\subsection{Asymmetrical Bridgman}

Figure 2 schematically depicts the principle of asymmetrical Bridgman. The experimental arrangement is similar to the conventional Bridgman except that the growth ampoule in this method is positioned asymmetrically instead of centrally aligning it to the furnace/baffle axis. By suitably placing an appropriate baffle in the furnace, it is possible to achieve quite a high vertical (axial) temperature gradient $\left(>50^{\circ} \mathrm{C} / \mathrm{cm}\right.$ ) along with a low horizontal (radial) gradient. The solid-liquid interface in this case, though inclined, becomes quite flat. To facilitate proper nucleation of seed the ampoule design is also suitably modified (figure $2 \mathrm{~b}$ ). The technique allows the use of larger ampoule diameters also (up to $21 \mathrm{~mm}$ dia crystals obtained).

The constant composition planes in Bridgman-grown crystals are expected to be parallel to the $s-1$ interface. But generally the delineation of $s-1$ interface in a grown ingot is an extremely cumbersome task, due to the variation in its inclination along the ingot axis. However, with judicious choice of furnace/ampoule design/positioning and growth parameters, it is possible to minimize the inclination/variation of s-1 interface to the ampoule axis without compromising on its flatness. Figure 3 compares the $s-1$ interface shapes in two cases, where the ingots being grown by asymmetrical Bridgman method under different conditions/positioning are quenched at an intermediate stage during the run. It can be seen that excellent $s-1$ interface flatness can be achieved along with a low axial inclination.

Figures $4 \mathrm{a}$ and $4 \mathrm{~b}$ compare the radial compositional profiles, as revealed by EPMA, across the asymmetrical Bridgman-grown crystals sliced parallel to the transverse and $s-1$ interface planes respectively. It can be seen that the crystal cut parallel to the $s-1$ interface possesses fairly uniform composition. In the transversely cut crystal, the 


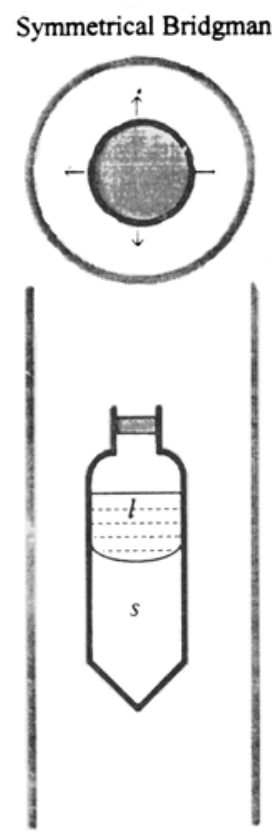

(a)

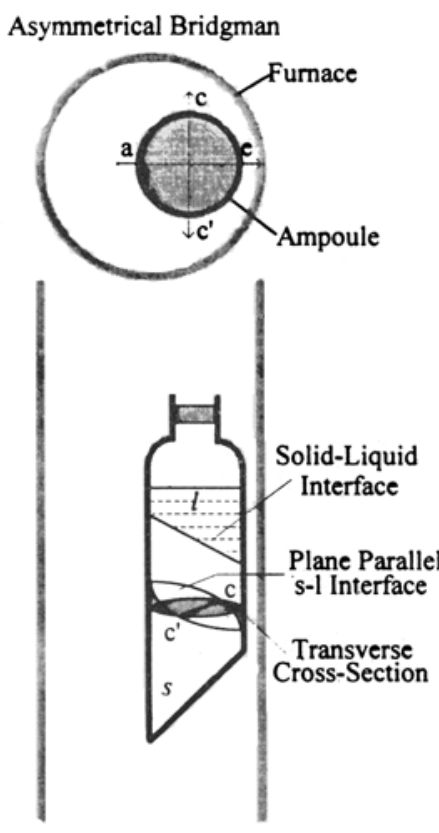

(b)

Figure 2. Schematic representation of ampoule position and shape of solid-liquid interface in (a) symmetrical and (b) asymmetrical Bridgman.

composition varies continuously from one end to the other along the line a-e, but in the perpendicular direction the same is nearly constant. These constant temperature lines are the intersections of $s-1$ interface planes with the transverse cross-sections as schematically depicted in figure $2 b$. For linear array fabrication, normally narrow (1-2 mm wide) strips of material are utilized. Such strips cut along $\mathrm{c}-\mathrm{c}^{\prime}$ direction possess excellent compositional uniformity and, in turn, would result in fairly uniform detector characteristics.

The technique of asymmetrical Bridgman though basically developed for MCT could also be successfully applied for growing good-quality crystals of other lowthermal-conductivity materials like CdTe, CdZnTe, CdTeSe, etc. It is applicable in case of horizontal Bridgman as well, although for MCT, particularly, the horizontal Bridgman is inapplicable due to the extremely high $\mathrm{Hg}$ vapour pressures involved.

\subsection{The horizontal casting of MCT for SSR growth}

The cast obtained in the first step of the SSR process contains microscopic inhomogeneities due to the dendritic freezing - three-dimensional dendrite trees immersed in an interdendritic matrix having a comparatively lower CdTe content. However, it is removed through the process of $\mathrm{Hg} / \mathrm{Cd}$ interdiffusion during the initial course of the second step of high-temperature long-term annealing. But the macroscopic compositional variations present in the cast as a result of non-uniform dendritic distributions etc are not affected appreciably. A very fine and fairly uniformly dendritic 


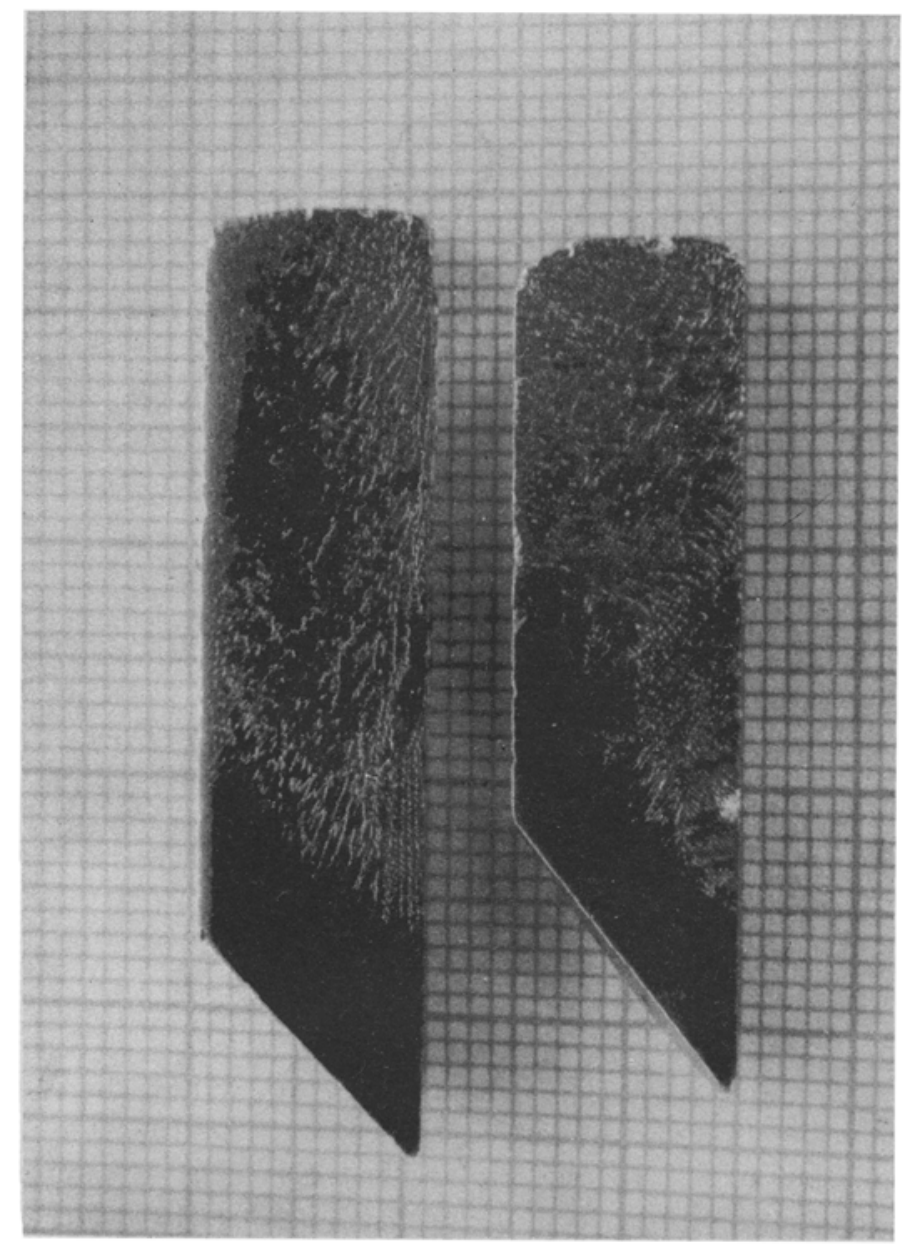

Figure 3. The shape of solid-liquid interface delineated in quenched ingots being grown by asymmetrical Bridgman under different conditions. The dendritic structure represents the melt.

distribution is therefore desirable in the cast in order to attain a high degree of compositional uniformity in the SSR-grown ingots.

The quenching of the melts for obtaining good-quality cast is normally done by forcing compressed air/gas on the sealed quartz ampoules while keeping the vapour space protected through thermal lagging to maintain the $\mathrm{Hg}$ vapour pressure for avoiding melt eruption. Figure 5 compares the casting procedure in vertical (conventional) and horizontal configurations. A vertically quenched cast normally contains a core region where the density of dendritic matrix becomes low due to the initial depletion of $\mathrm{CdTe}$ in the first-to-freeze peripheral region. On the other hand, the horizontally cast ingot is expected to possess a fairly uniform dendritic distribution as depicted. A large $s-1$ interface requires to move across the small thickness of the melt improving the quenching efficiency considerably, thereby resulting in a far better uniformity of composition compared to the conventionally obtained cast. However, obtaining a perfect cast free from pores/core/precipitates is technically a difficult task. 


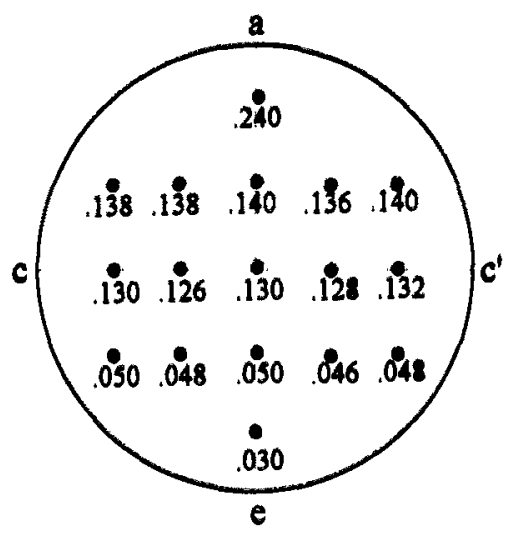

(a)

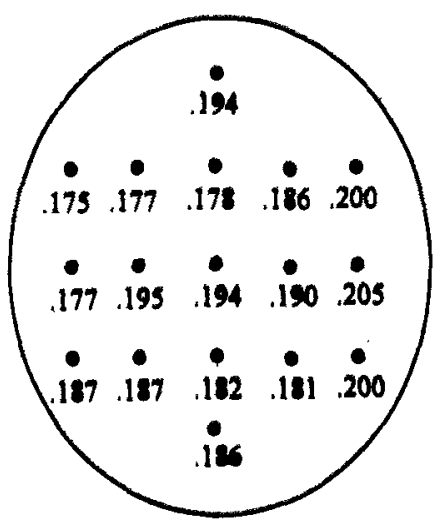

(b)

Figure 4. Compositional map ( $x$ value) of wafers cut parallel to (a) transverse cross-section and (b) solid-liquid interface.

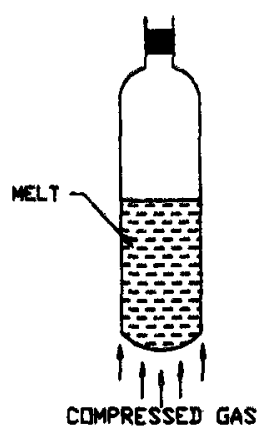

(a)

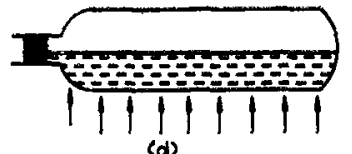

(d)

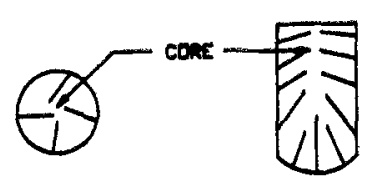

(b)

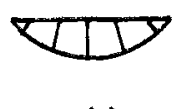

(e)

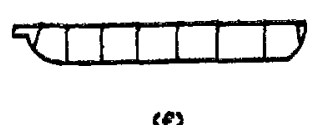

(p)

Figure 5. Schematic representation of (t) conventional and (d) horizontal casting configurations; (b), (e) and (c), (f) show expected dendritic distribution in transverse and longitudinal cross-sections for respective configurations.

After sustained experimentation the horizontal casting was first demonstrated with an arrangement in which the material-containing quartz ampoule was further sealed, under vacuum, in a thin-walled quartz ampoule with a definite amount of tin metal added to it. In horizontal configuration the molten tin partly filled the space between the two quartz tubes and provided a conducting path for the heat during the quenching, which was done by forcing compressed air on the lower part of the ampoule as schematically depicted in figure $5 \mathrm{~d}$. This resulted in excellent compositional uniformity in the grown ingots. As evident from the expected dendritic distribution the compositional uniformity was best in the longitudinal directions. Composition variation as good as $\Delta x \sim 0.001$ along $30 \mathrm{~mm}$ lengths in longitudinal 
directions and $\Delta x \sim \pm 0.002$ over the span of a $30 \times 15 \mathrm{~mm}^{2}$ area in longitudinally cut planks was achieved. These results compared well with those for even good-quality LPE layers. In addition to the excellent compositional uniformity, the crystalline perfection of these crystals was also found to be better. The core regions of the horizontally cast ingots are expected to be free of edge strains and, in fact, they were found to be free from subgrain structure. The dislocation (etch pit) densities in these regions were found to be about an order of magnitude lower compared to those normally obtained in conventionally cast SSR crystals.

Although the casting procedure described above was extremely effective and efficient it was technically a bit cumbersome due to the procedure of double wall sealing and determination of correct quantity of tin metal. A variation of this process was therefore evolved. The new experimental arrangement is schematically shown in figure 6. A semicircular stainless steel hollow jacket with end tubes for gas flow through it was designed for this purpose. The melt-containing ampoule rests horizontally on the matching internal grove of the jacket. The upper portion of the ampoule is carefully covered with thermal lagging. When compressed air is passed through the jacket, it quickly gets cooled and the heat is extracted through the lower peripheral region of

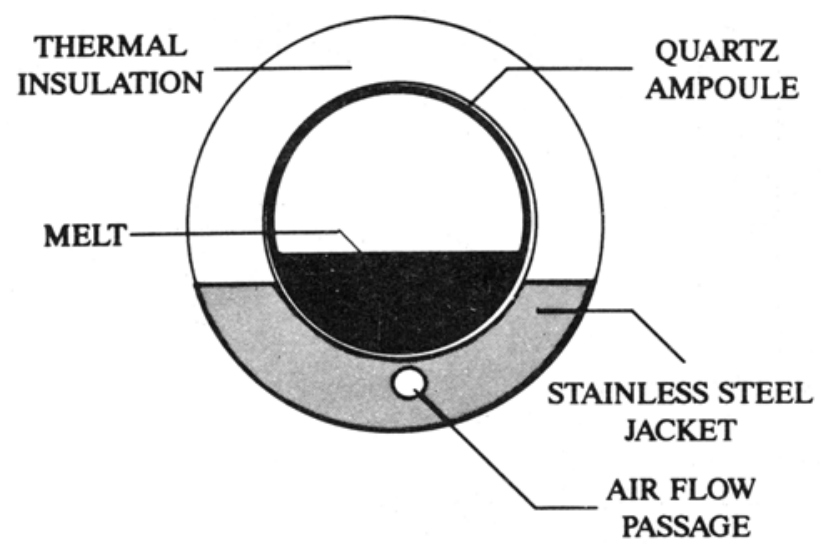

(a)

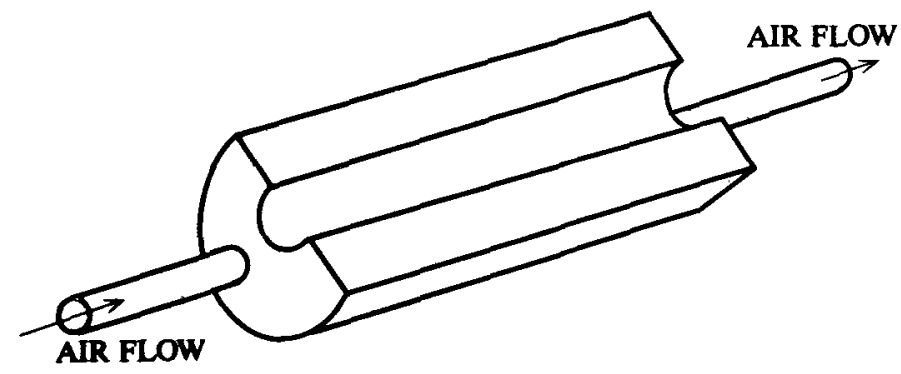

(b)

Figure 6. (a) Experimental arrangement for the horizontal casting and (b) schematic drawing of the casting apparatus. 


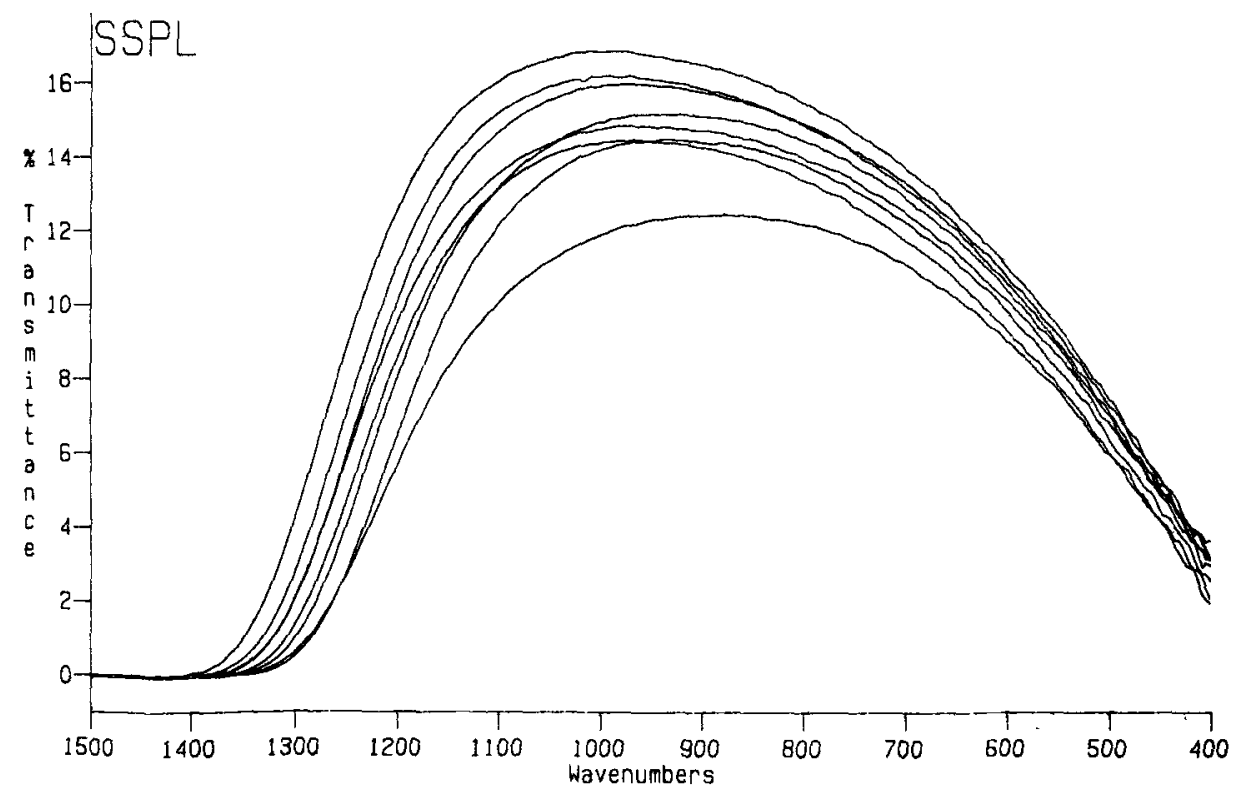

Figure 7. IR transmittance spectra of a $20 \times 15 \mathrm{~mm}^{2}$ longitudinally cut MCT plank, scanned across its area. The corresponding variation in composition, $\Delta x$, is of the order of. \pm 0.0025 .

the ampoule, thereby forcing a horizontal casting. By properly standardizing various associated parameters excellent casts could be obtained.

Figure 7 shows IR transmittance spectra across a $20 \times 15 \mathrm{~mm}^{2}$ plank, longitudinally cut from an SSR-grown crystal with the above arrangement. The compositional profile of this wafer deduced from the transmittance curves using the standard procedure (Micklethwaite 1988) reveals that the overall composition uniformity of this plank is almost of the same order as obtained in the initial experiment, the compositional variation being $\Delta x \sim \pm 0.0025$, and the linear variation along the longitudinal direction being $\Delta x \sim \pm 0.0015$ over $20 \mathrm{~mm}$. As described in case of asymmetrical Bridgman, excellent uniformity in spectral response could be achieved if the arrays are suitably aligned.

\section{Conclusions}

The asymmetrical Bridgman technique has been successfully applied to MCT bulk growth yielding crystals with fairly high compositional uniformity. The technique has the potential to solve the problem of acute curvature in solid-liquid interface in low-thermal-conductivity materials in general.

The horizontal casting procedure applied to the SSR growth of MCT improves the compositional uniformity, crystalline perfection and overall yield of crystals. Largesized $\left(\sim 20 \times 15 \mathrm{~mm}^{2}\right)$ wafers with compositional uniformity as good as $\Delta x \sim \pm 0.0025$ could be readily obtained using this process.

The excellent uniformity of composition along the longitudinal direction in horizontally cast SSR crystals and in specific transverse directions in asymmetrical 
Bridgman crystals could be utilized to fabricate linear IR detector arrays with improved spectral response uniformity.

\section{References}

Bagai R K and Borle W N 1989 J. Crystal Growth 94561

Capper P, Brice J C, Jones C L, Coates W G, Gosney J J G, Ard C and Kenworthy I 1988 J. Crystal Growth 89171

Cruse P W 1981 in Semiconductors and semimetals (eds) R K Willardson and R K Beer (New York: Academic Press) Vol. 18, p. 1

Elliot C T and Gordon N T 1993 in Handbook on semiconductors (eds) T S Moss and C Hilsum (Amsterdam: North Holland) Vol. 4, p. 841

Luigi Colombo, Syllaious A J, Perlakey R W and Brau M J 1985 J. Vacuum Sci. \& Technol. A3 95

Micklethwaite W F H 1981 in Semiconductors and semimetals (eds) R K Willardson and R K Beer (New York: Academic Press) Vol. 18, p. 48

Micklethwaite W F H 1988 J. Appl. Phys. 632382

Proc. SPIE Meeting 1979 San Diego, California 197

Sharma R K and Sharma B B 1987 Indian J. Phys. A61 350

Sharma R K, Singh V K, Nayyar N K, Gupta S R and Sharma B B 1993 J. Crystal Growth 131565

Vere A W, Straughan B W, Williams D J, Shaw N, Royale A, Gough J S and Mullin J B 1982 J. Crystal Growth 59121 